\title{
Análise perceptivo-auditiva da estabilidade vocal de adolescentes em diferentes tarefas fonatórias****
}

\author{
Perceptual analysis of adolescents’ vocal stability during different \\ phonation tasks
}

\author{
Michelle Ferreira Guimarães* \\ Mara Suzana Behlau** \\ Ivone Panhoca***
}

\begin{abstract}
*Fonoaudióloga. Doutoranda em Neurociências e Biologia Celular pela Universidade Federal do Pará. Coordenadora do Curso de Fonoaudiologia da Faculdade Seama (Macapá - AP). Endereço para correspondência: Av. Nações Unidas, 1201 - Macapá - AP - CEP 68908-126. (guima.michelle@gmail.com).

**Fonoaudióloga. Pós-Doutora pela University of California in San Francisco. Diretora do Centro de Estudos da Voz (CEV).

***Fonoaudióloga. Pós doutora pela University of Houston, Universidad de Salamanca e Washington University in Sant Louis. Docente do Curso de Graduação em Fonoaudiologia da Pontifícia Universidade Católica de Campinas.

****Trabalho Realizado no Departamento de Pediatria da Universidade Estadual de Campinas e no CEV. Dados Coletados em Escola Pública da Cidade de Campinas - SP.
\end{abstract}

Artigo Original de Pesquisa

Artigo Submetido a Avaliação por Pares

Conflito de Interesse: não

Recebido em 17.03.2010.

Revisado em 08.07.2010; 14.11.2010.

Aceito para Publicação em 30.11.2010.

\begin{abstract}
Background: quality and frequency variability of adolescents' voice, during puberty, in different phonation tasks. Aim: to analyze the vocal stability of adolescents in three different phonation tasks using a voice perceptual analysis. Method: participants were male students ( $n=46$ individuals), ranging in age from 13 to 15 years, from a public school in Campinas - SP. Voice samples were recorded using a digital recorder in three different tasks: speaking a sustained vowel /a/, counting from one to ten, and reading. Three voice specialists evaluated stability by means of voice perceptual analysis. For the voice samples that were considered unstable, the Analogical Visual Scale (AVS) of $10 \mathrm{~cm}$ was used to estimate the instability level, where zero means absence of instability and ten refers to maximum instability. Results: $78.3 \%$ of the adolescents presented vocal instability when speaking the sustained vowel, varying from one to nine in the AVS. Only one adolescent presented unstable voice when counting numbers (level =1). Vocal instability was not observed during reading for any of the participants. Vocal stability varied significantly among phonation tasks and adolescents showed greater instability during the sustained vowel $(\mathrm{p}<0,0001$; g.l = 2). Conclusion: counting numbers and reading do not detect vocal instability; the production of a sustained vowel demonstrated to be a better task.
\end{abstract}

Key Words: Adolescent; Voice Quality; Puberty.

\section{Resumo}

Tema: variabilidade de qualidade e frequência da voz de adolescentes, durante a puberdade, em diferentes tarefas fonatórias. Objetivo: analisar, por meio da avaliação perceptivo-auditiva, a estabilidade vocal de adolescentes em três diferentes tarefas fonatórias. Método: foram sujeitos do estudo 46 adolescentes do sexo masculino, com idade entre 13 e 15 anos, estudantes de uma escola estadual da cidade de Campinas - SP, onde foi realizada a coleta dos dados. As vozes foram gravadas em gravador digital, solicitou-se emissão sustentada da vogal /a/, contagem de 1 a 10 e leitura de parágrafo de um livro pré-estabelecido. A avaliação perceptivo-auditiva da estabilidade vocal foi realizada por três fonoaudiólogas especialistas em voz. Para vozes consideradas instáveis, utilizou-se uma Escala Visual Analógica de 10cm para marcação do grau de instabilidade, em que 0 significava estabilidade e 10 instabilidade máxima, podendo variar de 0 a 10. Resultados: na emissão da vogal sustentada 78,3\% $(n=36)$ dos adolescentes apresentaram instabilidade vocal, em graus que variaram de 1 a 9 . Apenas um adolescente apresentou voz instável, classificada como grau 1, durante a contagem de números e todos apresentaram voz estável durante a leitura. A ocorrência de estabilidade variou significativamente de acordo com a tarefa fonatória, havendo maior instabilidade na emissão de vogal sustentada ( $<0,0001$; g.l = 2). Conclusão: as tarefas fonatórias de contagem de números e leitura não permitem inferir sobre a presença de instabilidade na emissão, o que deve ser avaliado na vogal sustentada.

Palavras-Chave: Adolescente; Qualidade da Voz; Puberdade; Voz

Referenciar este material como:

1 Guimarães MF, Behlau MS, Panhoca I. Análise perceptivo-auditiva da estabilidade vocal de adolescentes em diferentes tarefas fonatórias. Pró-Fono Revista de Atualização Científica. 2010 out-dez;22(4):455-8. 


\section{Introdução}

A voz é tipicamente variável em qualidade e frequência durante o período da puberdade ${ }^{1}$. Adolescentes geralmente apresentam fases de instabilidade vocal principalmente durante a muda vocal $^{2}$. A muda vocal pode ser definida como um conjunto de mudanças no padrão de voz que ocorre entre a infância e a puberdade ${ }^{3}$. A instabilidade da emissão pode estar associada, também, a determinadas faixas de peso e ganho de peso rápido ${ }^{4}$.

O nível de testosterona no sangue, a velocidade de crescimento e o grau de desenvolvimento puberal são rotineiramente usados para prever o início da mutação fisiológica ou estabelecer quando ela ocorreu ${ }^{5-6}$. As mudanças vocais na adolescência devem ser avaliadas por meio de determinados parâmetros perceptivoauditivos e acústicos, com o propósito de verificar os efeitos do processo da muda vocal na $\mathrm{voz}^{7-8}$.

A voz durante a muda pode ser dividida em três fases: a pré-muda, fase que antecede a ocorrência da muda vocal; a muda vocal em si, especificada pelo período de instabilidade vocal; e a pós-muda, quando o adolescente já apresenta qualidade vocal adulta ${ }^{2}$. As mudanças vocais mais evidentes em adolescentes do sexo masculino ocorrem dos 13 aos 15 anos de idade ${ }^{9}$, sendo elas: redução da frequência fundamental, predomínio do registro de peito, instabilidade da emissão, qualidade vocal rouca, diplofônica, áspera e/ou soprosa $^{10}$. A muda pode durar de 6 meses a 1 ano e alguns adultos lembram-se da fase de muda principalmente pela presença de flutuações vocais $^{11}$.

O objetivo do estudo foi analisar, por meio de avaliação perceptivo-auditiva da voz, a estabilidade vocal, em três diferentes tarefas fonatórias, de adolescentes do sexo masculino durante a puberdade.

\section{Método}

O presente estudo foi aprovado pelo Comitê de Ética em Pesquisa da Faculdade de Ciências Médicas da Universidade Estadual de Campinas (protocolo número 570/2003).

Foram sujeitos desse estudo, 46 adolescentes do sexo masculino de uma amostra de conveniência, na faixa etária de 13 a 15 anos, estudantes de sexta, sétima e oitava séries do Ensino Fundamental de uma escola pública, localizada na cidade de
Campinas. A diretora, representante da instituição, assinou o Termo de Consentimento Livre e Esclarecido concordando com a realização da pesquisa, os objetivos do presente estudo foram esclarecidos e os alunos foram convidados a participar do projeto. Os responsáveis e os alunos também assinaram e o Termo de Consentimento Livre e Esclarecido.

Foram incluídos na pesquisa adolescentes do sexo masculino com idade entre 13 e 15 anos e excluídos os adolescentes que apresentassem queixas vocais, problemas neurológicos, síndromes congênitas, afecções de vias aéreas superiores e/ou inferiores ou que utilizassem medicamentos para controlá-las.

As vozes dos adolescentes foram gravadas em gravador digital Panasonic RR-US360® em sala da própria escola com ruído ambiental inferior a 50 dB. Os jovens foram orientados a emitir, em pé, a vogal /a/ sustentada em sua intensidade habitual; falar os números de 1 a 10 e ler parágrafo de um livro pré-estabelecido. Foi utilizado microfone PHILIPS SBC MD680®, unidirecional, posicionado com distância de 5 cm da boca para gravação da vogal sustentada, e $10 \mathrm{~cm}$ para gravação da fala encadeada, mantendo-se uma angulação de 45 graus a fim de reduzir o ruído aerodinâmico da articulação ${ }^{12-13}$.

As análises dos parâmetros vocais estabilidade e instabilidade foram realizadas por três fonoaudiólogas especialistas em voz, por meio de análise perceptivo-auditiva. Foi utilizada uma Escala Visual Analógica (EVA) de 10cm, em uma linha reta, para marcação do grau de instabilidade, em que uma extremidade foi representada por 0 (zero) indicando estabilidade vocal e a outra por 10 (dez) indicando instabilidade máxima. O grau de instabilidade variou de 1 a 10 de acordo com os centímetros previamente marcados.

Das amostras da vogal /a/ foram excluídos o início e o término das emissões, por geralmente apresentarem características irregulares. O tempo das amostras foi padronizado em três segundos.

A análise da estabilidade e instabilidade nas diferentes tarefas fonatórias foi realizada através de estatística descritiva de médias, valor mínimo e máximo. O mesmo procedimento foi utilizado para análise do grau de instabilidade nas diferentes tarefas. Para verificar se a instabilidade vocal diferiu nas diferentes tarefas fonatórias foi utilizado o teste Chi-Quadrado de Pearson, por meio do programa John's Macintosh Project (JMP) do Statical Analysis System (SAS) Institute. Foi adotado nível de significância igual a 0,05. 


\section{Resultados}

A instabilidade vocal ocorreu em $78,3 \%$ dos adolescentes estudados durante a tarefa de emissão sustentada da vogal /a/ (Tabela 1). O grau de instabilidade variou de 1 a 9 (Tabela 2).

A maioria dos adolescentes que apresentaram instabilidade na emissão da vogal sustentada /a/ (36,1\%) foram classificados em grau 1 da EVA, o que pode ser considerado uma instabilidade discreta, seguidos pelos graus 2 (16,6\%) e 4 $(16,6 \%)$. Observou-se que 4 adolescentes $(11,1 \%)$ foram classificados como grau 9, ou seja, apresentaram instabilidade de emissão próxima à instabilidade considerada máxima pelo avaliador.
Apenas 3 adolescentes (8,3\%) foram classificados em grau 3, 2 adolescentes (5,5\%) em grau 6 e um adolescente $(2,7 \%)$ em graus 5 e 7 . Não houve adolescentes classificados em graus 8 ou 10 (Tabela2).

Na tarefa fonatória de contagem de números, dos 46 adolescentes avaliados, apenas 1 (2,2\%) apresentou instabilidade à emissão, considerada como grau 1, ou seja, instabilidade discreta (Tabela 1).

Na tarefa fonatória de leitura de parágrafo de um livro pré-estabelecido todos os adolescentes (100\%) apresentaram emissão estável (Tabela 1).

Por meio do teste Chi-quadrado de Pearson observou-se que a ocorrência de_estabilidade variou significantemente entre as diferentes tarefas e houve maior instabilidade na tarefa de vogal sustentada do que nas demais $(\mathrm{p}<0,0001$; g.l $\mathrm{l}=2)$.

TABELA 1. Distribuição numérica e percentual da análise perceptivo-auditiva da estabilidade e instabilidade vocal nas diferentes tarefas fonatórias.

\begin{tabular}{|c|c|c|c|c|c|c|c|}
\hline \multirow{2}{*}{ Tarefas } & \multicolumn{2}{|c|}{ Estável } & \multicolumn{2}{|c|}{ Instável } & \multirow{2}{*}{ Grau Mínimo } & \multirow{2}{*}{ Média Grau } & \multirow[t]{2}{*}{ Grau Máximo } \\
\hline & $\mathrm{N}$ & $\%$ & $\mathrm{~N}$ & $\%$ & & & \\
\hline vogal /a/ sustentada & 10 & 21,7 & 36 & 78,3 & 1 & 3,27 & 9 \\
\hline contagem & 45 & 97,8 & 1 & 2,2 & 1 & 1 & 1 \\
\hline leitura & 46 & 100 & 0 & 0 & 0 & 0 & 0 \\
\hline
\end{tabular}

TABELA 2. Distribuição numérica e percentual de indivíduos com instabilidade vocal e seus graus de instabilidade na emissão da vogal sustentada /a/.

\begin{tabular}{c|c|c}
\hline Grau de Instabilidade & N & $\%$ \\
\hline 1 & 13 & 36,1 \\
2 & 6 & 16,6 \\
3 & 3 & 8,3 \\
4 & 6 & 16,6 \\
5 & 1 & 2,7 \\
6 & 2 & 5,5 \\
7 & 1 & 2,7 \\
8 & 0 & 0 \\
9 & 4 & 11,1 \\
10 & 0 & 0 \\
\hline
\end{tabular}

\section{Discussão}

Durante a adolescência ocorre a transição gradual entre a infância e a idade adulta ${ }^{14}$. Desta forma, uma adaptação às novas condições anatômicas torna-se necessária ${ }^{15}$. No que se refere à voz, essa adaptação leva um período de alguns meses a um ano $^{10} \mathrm{e}$ a mutação vocal, assim como o som da voz e as características típicas da voz nesse período, estão relacionados ao crescimento da laringe, à variação do comprimento e espessura das pregas vocais ${ }^{16-17}$, mudança nos caracteres sexuais secundários ${ }^{18-19}$ e o índice de massa corporal ${ }^{20}$.

O conhecimento detalhado do desenvolvimento vocal normal é um pré-requisito para o diagnóstico e tratamento das disfunções vocais entre crianças e adolescentes por meio de métodos adequados para sua idade e seus estágios de desenvolvimento ${ }^{16}$.

Em nosso estudo observamos que a emissão da vogal sustentada evidenciou claramente a instabilidade vocal e, portanto, essa tarefa pode e deve ser utilizada para investigar possíveis alterações vocais ${ }^{21-22}$, e a habilidade do indivíduo em controlar as forças aerodinâmicas e mioelásticas da laringe ${ }^{23}$.

A contagem de números oferece dados sobre a habilidade do indivíduo em controlar a respiração e a fonação durante a fala ${ }^{17}$. Desta forma, pode-se inferir, baseado em nossos resultados, que a maioria 
dos adolescentes estudados $(97,8 \%)$ apresenta eficiência glótica adequada.

Já em relação à tarefa de leitura de um parágrafo de texto pré-estabelecido evidenciamos a estabilidade de emissão em todos os indivíduos estudados. Boone e McFarlane ${ }^{17}$; Colton e Casper ${ }^{24}$ consideram importante a leitura de um texto padrão para avaliação da voz, porém é importante utilizar diferentes tipos de tarefas fonatórias para a avaliação perceptivoauditiva da $\mathrm{voz}^{25-26}$, principalmente para avaliar a estabilidade da emissão, uma vez que a alteração desse parâmetro pode passar despercebida em tarefas de fala automática e encadeada.

Embora a avaliação vocal necessite de diferentes tarefas fonatórias para se obter dados

\section{Referências Bibliográficas}

1. Raj A, Gupta B, Chowdhury A, Chadha S. A study of voice changes in various phases of menstrual cycle and in postmenopausal women. Journal of Voice. 2010; 24(3):363-8.

2. Fuchs M, Fröehlich M, Hentschel B, Stuermer IW, Kruse E, Knauft D. Predicting mutation change in the speaking voice of boys. Journal of Voice. 2007; 21(2):169-78.

3. Santos MA, Moura JM, Duprat Ade C, Costa HO, Azevedo $\mathrm{BB}$. The interference of voice change on structural vocal cords lesions. Braz J Otorhinolaryngol. 2007;73(2): 226-30.

4. Willis EC, Kenny DT. Relationship between weight, speaking fundamental frequency, and the appearance of phonational gaps in the adolescent male changing voice. Journal of Voice. 2008;22(4):451-71.

5. Fuchs M, Behrendt W, Keller E, Kratzsch J. Methods for prediction of the beginning of mutation in boys voices: investigations in singers of the Thomaner Choir Leipzig. Folia Phoniatr Logop. 1999;51:261-71.

6. Fuchs M, Behrendt W, Kratzsch J, Keller E. Forecast of voice mutation at singers of professional boy's choirs with parameters of growth and puberty, insulin-like growth factor I and testosterone - investigation on singers of the Thomanerchor Leipzig. Horm Res. 1997;48:133.

7. Bonet M, Casan P. Evaluation of dysphonia in a children's choir. Folia Phoniatr Logop. 1994;46:28.

8. Baken RJ, Orlikoff RF. Voice measurement: is more better? Log Phon Vocol. 1997;22:147-51.

9. Zhu JL, Basso O, Obel C, Bech BH, Nohr EA, Shrestha A, Olsen J. Parental infertility and sexual maturation in children. Hum Reprod. 2009;24(2):445-50.

10. Behlau MS, Azevedo R, Pontes P. Conceito de voz normal e classificação das disfonias. In: Behlau MS. Voz: O livro do especialista. Rio de Janeiro: Revinter. 2001. p. 54-76.

11. Gil D, Lourenço L, Miranda AR, Pereira AJ, Rodrigues S, Behlau MS. A memória da muda vocal. Acta AWHO. 1994;13:74-80.

12. Price DB, Sataloff RT. A simple technique for consistent fidedignos quanto à qualidade vocal do indivíduo, a tarefa de emissão de vogal sustentada foi a que evidenciou alteração da estabilidade vocal.

\section{Conclusão}

A análise de vozes de adolescentes entre 13 e 15 anos permitiu concluir que a vogal sustentada revela as instabilidades vocais típicas do período da muda vocal, não reveladas nas tarefas de fala encadeada, quer seja na fala automática (contagem de números), quer seja na leitura de um texto. A instabilidade, quando presente, foi discreta, na maioria dos casos analisados.

microphone placement in voice recording. Journal of Voice. 1988;2:206-7.

13. Titze IR. Principles of voice production. Prentice Hall: Englewood Cliffs; 1994.

14. Colli AS. Conceito de adolescência. In: Marcondes E. Pediatria Básica. São Paulo: Savier; 1994. p. 474-500.

15. Zemlin WR. Speech and hearing science, anatomy and physiology. Prentice Hall: Englewood Cliffs; 1968.

16. Fuchs M. Landmarks of physiological development of the voice in childhood and adolescense. Laryngorhinootologie. 2008; 87(1):10-6.

17. Boone D, McFarlane SC. A voz e a terapia vocal. 5aedição. Porto Alegre: Artes Médicas; 1994.

18. Bredant, TCM. Alterações endócrinas e suas implicações vocais no período da adolescência [Monografia]. Curitiba: Centro de Especialização em Fonoaudiologia Clínica; 1999.

19. Charpy N. La meu des adolescentes. Rev Laryngol Otol Rhynol (Bord). 2002; 123(5):297-301.

20. Juul A, Magnusdottir S, Scheike T, Prytz S, Skakkebaek NE. Age at voice break in Danish boys: effects of prepubertal body mass index and secular trend. Int J Androl. 2007; 30(6):537-42.

21. Cardoso APR. Análise perceptivo-auditiva da qualidade vocal nas tarefas de vogal sustentada e fala encadeada em diferentes tipos de disfonia [Monografia]. São Paulo(SP): Centro de Estudos da Voz; 2005.

22. Fex S. Perceptual evaluation. Journal of Voice. 1992;6:155-8.

23. VonLeden H, Koike Y. Detection of laryngeal disease by computer technique. Arch Otolaryngol. 1970;91:3-10.

24. Colton RH, Casper JK, Leonard R. Understanding voice problems: a physiological perspective for diagnoses and treatment. Philadelphia: Lippincott Williams \& Wilkins; 2006.

25. Sonninem AH, Hurne P. On the terminology of voice research. Journal of Voice. 1992; 6:188-293.

26. Hammarberg B, Fritzell B, Gauffin J, Sundberg J, Wedin L. Perceptual and acoustic correlates of abnormal voice quality. Acta Otolaryngol. 1980; 90:441-51. 